\title{
Detecting Mechanochemical Atropisomerization within an STM Break Junction
}

Edmund Leary, $, \#, \dagger \odot$ Cécile Roche, ${ }^{\ddagger}$ Hua-Wei Jiang, ${ }^{\ddagger}$ Iain Grace, ${ }^{\S}$ M. Teresa González," Gabino Rubio-Bollinger, ${ }^{\perp}$ Carlos Romero-Muñiz, ${ }^{\square}$ Yaoyao Xiong, ${ }^{\ddagger}$ Qusiy Al-Galiby,, ,I Mohammed Noori, ${ }^{\S, \infty}$ Maria A. Lebedeva, $\nabla \odot$ Kyriakos Porfyrakis, $\nabla$ Nicolás Agrait, Andrew Hodgson, ${ }^{\#, \dagger}$ Simon J. Higgins, ${ }^{\dagger}$ Colin J. Lambert, ${ }^{*},{ }^{\S}$ Harry L. Anderson, ${ }^{*}$, and Richard J. Nichols $*, \#, \dagger$ (1)

${ }^{\dagger}$ Department of Chemistry, Donnan and Robert Robinson Laboratories, University of Liverpool, Liverpool L69 7ZD, U.K.

${ }^{\#}$ Surface Science Research Centre and Department of Chemistry, University of Liverpool, Oxford Street, Liverpool L69 3BX, U.K.

‡Chemistry Research Laboratory, Department of Chemistry, Oxford University, Oxford OX1 3TA, U.K.

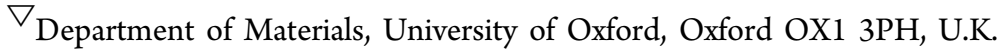

${ }^{\S}$ Department of Physics, Lancaster University, Lancaster, U.K.

"Instituto Madrileño de Estudios Advanzados (IMDEA), Calle Faraday 9, Campus Universitario de Cantoblanco, 28049 Madrid, Spain

${ }^{\perp}$ Departamento de Física de la Materia Condensada, and Instituto "Nicolás Cabrera" and $\square$ Departamento de Física Teórica de la Materia Condensada, Universidad Autónoma de Madrid, E-28049, Madrid, Spain

IIDepartment of Physics, College of Education, University of Al-Qadisiyah, 58002 Iraq

${ }^{\infty}$ Department of Physics, Collage of Science, Thi-Qar University, Thi-Qar 00964, Iraq

\section{Supporting Information}

ABSTRACT: We have employed the scanning tunneling microscope break-junction technique to investigate the single-molecule conductance of a family of 5,15-diaryl porphyrins bearing thioacetyl (SAc) or methylsulfide (SMe) binding groups at the ortho position of the phenyl rings (S2 compounds). These ortho substituents lead to two atropisomers, cis and trans, for each compound, which do not interconvert in solution under ambient conditions; even at high temperatures, isomerization takes several hours (half-life $15 \mathrm{~h}$ at $140{ }^{\circ} \mathrm{C}$ for SAc in $\mathrm{C}_{2} \mathrm{Cl}_{4} \mathrm{D}_{2}$ ). All the $\mathrm{S} 2$ compounds exhibit two conductance groups, and comparison with a monothiolated (S1) compound shows the higher group arises from a direct $\mathrm{Au}-$ porphyrin interaction. The lower conductance group is associated with the S-to-S pathway. When the binding group is $\mathrm{SMe}$, the difference in junction length distribution reflects the difference in $S-S$ distance $(0.3 \mathrm{~nm})$ between the

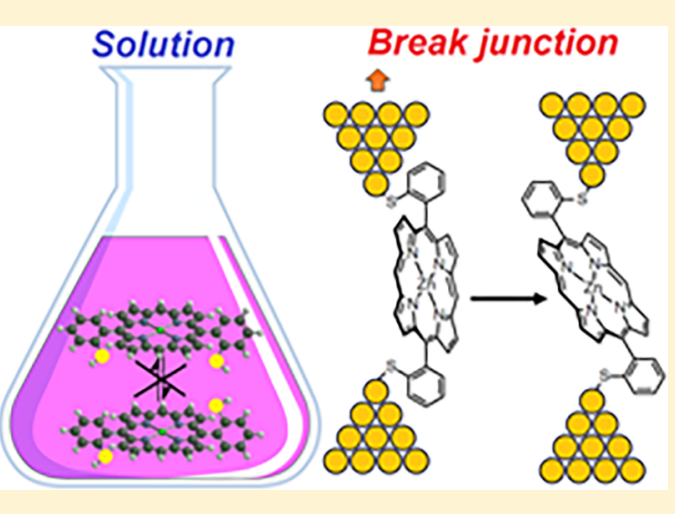
two isomers. In the case of SAc, there are no significant differences between the plateau length distributions of the two isomers, and both show maximal stretching distances well exceeding their calculated junction lengths. Contact deformation accounts for part of the extra length, but the results indicate that cis-to-trans conversion takes place in the junction for the cis isomer. The barrier to atropisomerization is lower than the strength of the thiolate $\mathrm{Au}-\mathrm{S}$ and $\mathrm{Au}-\mathrm{Au}$ bonds, but higher than that of the $\mathrm{Au}-$ SMe bond, which explains why the strain in the junction only induces isomerization in the SAc compound.

\section{INTRODUCTION}

Molecular electronics is an extremely exciting area of nanotechnology, which aims to develop new electronic devices operating at the single molecule level. ${ }^{1-4}$ Understanding and controlling the conformation of molecules in single molecule junctions (SMJs) is a key challenge on the road to functional electronic devices based on individual molecules. It is an important consideration for 'rigid' molecules, such as those based on conjugated $\pi$-systems ${ }^{5,6}$ and also for biomolecules with weaker internal hydrogen bonds ${ }^{7}$ such as DNA. ${ }^{8}$ Here, by studying a family of porphyrin compounds in which we specifically vary the anchor groups and conduct a detailed plateau-length analysis, we show it is possible to probe the conformation of the molecules between a pair of nanoelectrodes and moreover show that atropisomerization takes place due to the strain imposed on the molecules during tip retraction.

Received: October 10, 2017

Published: December 20, 2017 
Porphyrins are a class of organic heterocycles that garner much attention in the fields of nanoscience and molecular electronics. They have extensive $\pi$-conjugation and strong optical absorption in the visible spectrum, leading to prominent applications in light-harvesting systems ${ }^{9}$ and dye-sensitized solar cells. ${ }^{10}$ To study how charge transfer takes place at the level of individual molecules, photophysical experiments such as transient absorption spectroscopy have been used, ${ }^{11}$ but wiring individual molecules between a pair of electrodes and studying the conductance under an applied electrical bias should lead to new insights and potential new applications. ${ }^{12,13}$ There are a growing number of experiments on individually wired porphyrins using $S T M^{14,15}$ and break-junction (BJ)-based methods. ${ }^{16-18}$ The development of such single-molecule conductance techniques allows, in particular, the conductance, ${ }^{19,13} I V$ behavior, ${ }^{20}$ and thermopower ${ }^{21,22}$ to be probed at the single-molecule level. Previous studies on porphyrin singlemolecule junctions have investigated factors such as number of oligomer units, ${ }^{23,15}$ the influence of the central ion, ${ }^{16}$ the type of binding group,,$^{24,25}$ and also its position ${ }^{26,27}$ and image charge effects. ${ }^{28}$

The challenge of relating the conductance vs distance $\left(G_{z}\right)$ traces from break-junction (BJ) experiments is particularly important for molecules with large $\pi$-systems, such as porphyrins. With their extended $\pi$-surface, they have the potential to interact with gold electrodes in several ways, either solely through the anchor groups placed at the ends of the molecule, or via the face of the $\pi$-system. ${ }^{29}$ Such $\mathrm{Au}-\pi$ interactions, which have been explored previously in for example oligoenes, ${ }^{30}$ oligo(phenylene ethynylene)s, ${ }^{29,6}$ and fullerenes, ${ }^{31}$ are effectively considered to "short-circuit" the wire relative to the end-to-end pathway. It is therefore of great importance to know how molecules orient, and hence interact with, the electrodes across different junctions, in order to relate their conductance properties with structurally different compounds. The initial objective of the work presented here was to understand the interaction of sulfur-substituted porphyrins with gold electrodes, so as to measure charge transport through larger porphyrin nanorings. ${ }^{32}$

A few previous studies on porphyrins have shown examples of $G_{z}$ profiles in the form of $2 \mathrm{D}$-histograms, ${ }^{16}$ but there is a lack of extensive investigations of this type for porphyrins. We synthesized and isolated different conformational isomers of 5,15-diarylporphyrins bearing either thioacetate (RSAc) binding groups, which form thiolate (RS) junctions with gold, or methyl sulfide (RSMe). These anchor groups are attached to the aryl rings at the ortho position (relative to the porphyrin group) which gives rise to two possible stereoisomers, a cis and a trans form, due to hindered rotation around the phenylporphyrin bond (Figure 1). Our initial hypothesis was that during junction elongation, the $G_{z}$ profile would differ noticeably between the isomers due to different preferred geometries of the molecules within the junction. We also predicted that the average junction breakdown distance should differ by about $0.3 \mathrm{~nm}$, that is by the difference in $S$ to $S$ distance between cis and trans configurations.

Our main findings are as follows. Two distinctive conductance groups were found for all S2 compounds, and we assign the highest (G1) to a direct $\mathrm{Au}$-porphyrin interaction. This was confirmed by testing a monothiolated (S1) compound, which showed only high-conductance plateaus, similar to G1 of the S2. We thus associate the lower conductance events (G2) with a predominantly S-to-S
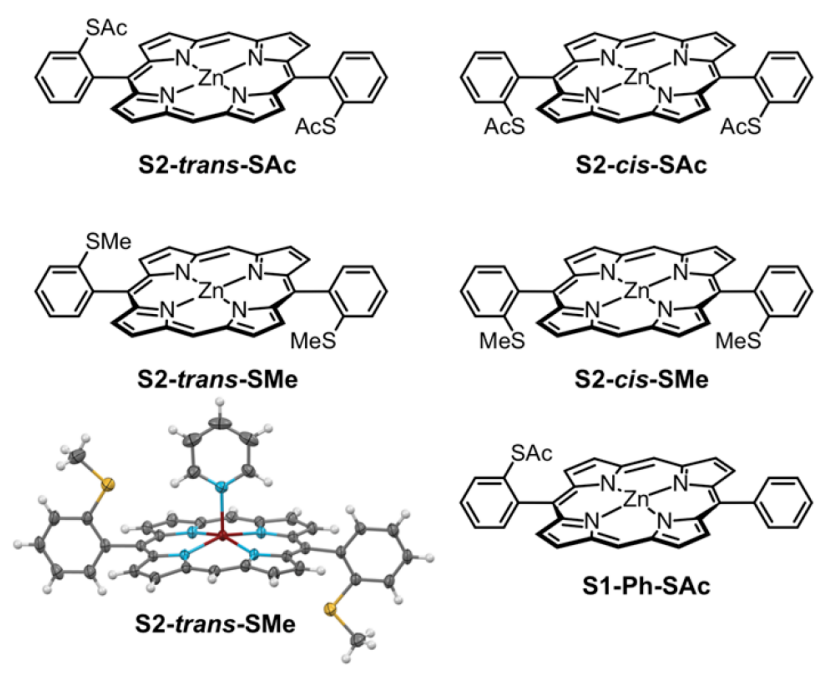

Figure 1. Structures of the compounds investigated, and X-ray structure of S2-trans-SMe with coordinated pyridine (yellow, S; red, $\mathrm{Zn}$; gray, $\mathrm{C}$; light blue, $\mathrm{N}$; white, $\mathrm{H}$ ).

conductance pathway. For the low-conductance plateaus, the initial hypothesis of shorter plateaus for the cis isomer was confirmed for the SMe-terminated compounds. This was not, surprisingly, the case for SAc, where we found no appreciable difference between cis and trans isomers in all runs. For SMe, all junction lengths were less than the calculated values, whereas for SAc we found that plateaus could exceed this length by $5-8$ $\AA$. To explain this behavior, we have to take into account the $\mathrm{Au}-\mathrm{S}$ binding energy, which is strong enough not only to deform the electrodes but also to force the isomerization of the cis to the trans configuration. The $\mathrm{Au}-\mathrm{SMe}$ binding energy is too low to affect these processes.

\section{EXPERIMENTAL METHODS}

Synthesis. Detailed synthetic routes and procedures are presented in Supporting Information (SI). The S2 compounds were obtained by condensation of dipyrromethane with SAc or SMe ortho-substituted benzaldehydes, followed by metalation with zinc(II) acetate. This procedure yielded a mixture of cis and trans atropisomers, S2-cis-SAc and S2-trans-SAc, and S2-cis-SMe and S2-trans-SMe, respectively. The isomers were separated by HPLC and are stable at room temperature. The cis and trans isomers have very similar ${ }^{1} \mathrm{H}$ NMR spectra with identical coupling patterns and very small differences in chemical shifts. A preliminary assignment was obtained by comparing their retention times on HPLC (normal phase). The cis isomer is expected to have a larger dipole moment than the trans and was therefore assigned as the product with the longer retention time. For the $\mathrm{SMe}$ isomers the assignment was confirmed by $\mathrm{X}$-ray crystallography on the isolated trans isomer (Figure 1). For the SAc isomers, the following experiment was performed to confirm the assignment: Starting from a strapped porphyrin ${ }^{32}$ with both thiol groups tethered into a cis arrangement by a bis-thioester linker, the strap was cleaved by addition of methylamine, and the resulting thiol groups were acylated to SAc with acetic anhydride. A single isomer, corresponding to the slower-running isomer on HPLC, was formed, thereby confirming the assignment of the cis isomer.

The monofunctionalized reference compound S1-Ph-SAc was synthesized by condensation of dipyrromethane with a mixture of unsubstituted benzaldehyde and SAc ortho-substituted benzaldehyde. The four porphyrin products formed in this reaction (unsubstituted 5,15-diphenylporphyrin, the desired monofunctionalized product, and the pair of bis-functionalized cis and trans atropisomers) were easily separated by column chromatography, and the monofunctionalized product was metalated to give S1-Ph-SAc. 


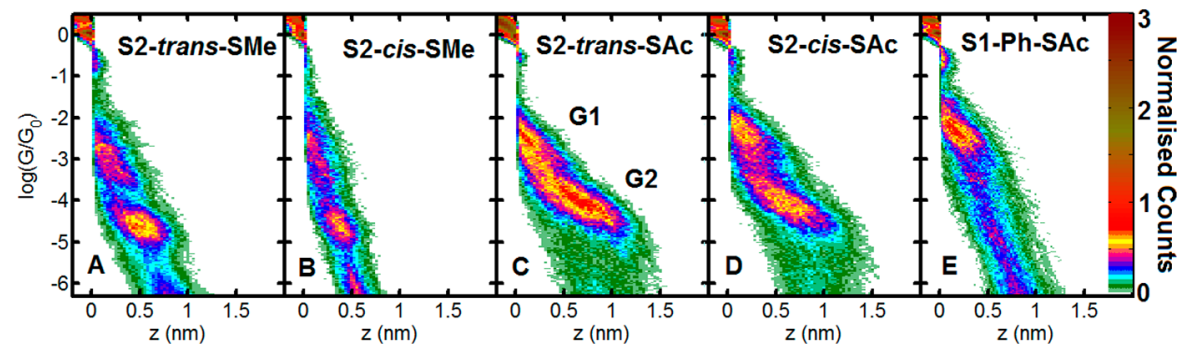

Figure 2. 2D histograms of the "plateau-containing" traces recorded using a tip-sample bias of $0.23 \mathrm{~V}$. The number of traces in each histogram with the percentage of the total as follows: A: 1288 (10.5\%), B: 510 (7.5\%), C: 1199(13.4\%), D: 1271 (9.3\%), E: 1186 (19.5\%).

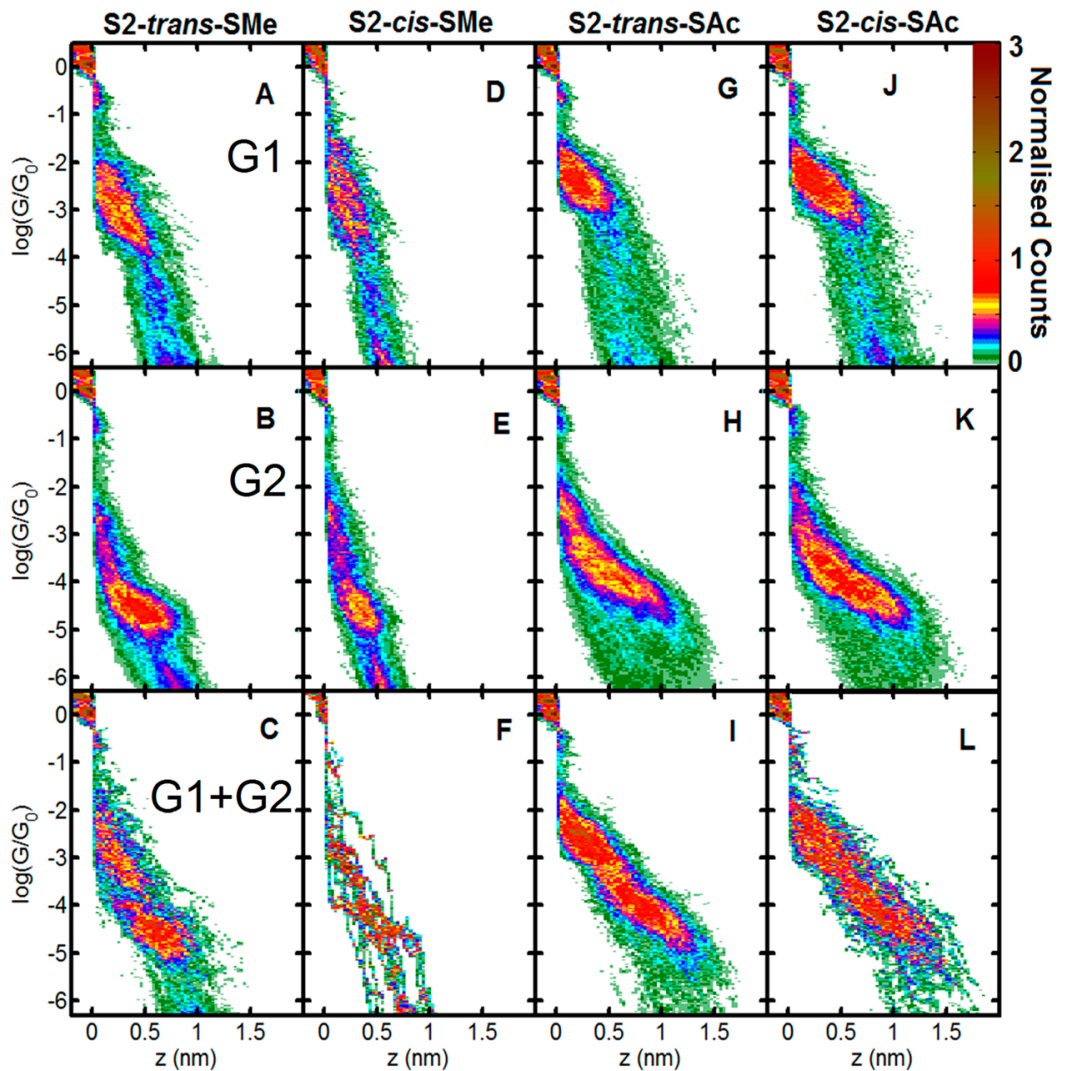

Figure 3. 2D histograms of the traces separated into various groups for each compound. (A) S2-trans-SMe, G1; (B) S2-trans-SMe, G2; (C) S2trans-SMe, G1and G2; (D) S2-cis-SMe, G1; (E) S2-cis-SMe, G2; (F) S2-cis-SMe, G1and G2; (G) S2-trans-SAc, G1; (H) S2-trans-SAc, G2; (I) S2trans-SAc, G1and G2; (J) S2-cis-SAc, G1; (K) S2-cis-SAc, G2; (L) S2-cis-SAc, G1and G2; where G1 refers to the high conductance and G2 the low conductance plateau.

Break-Junction Experiments. To form molecular junctions, we used the break-junction method as described previously with a homebuilt scanning tunneling microscope (STM). ${ }^{33}$ For details of sample preparation and further methodology, see SI. Briefly, a gold STM tip is repeatedly driven in and out of contact with a gold surface and the current monitored as a function of the distance traveled. At values close to $1 G_{0}$ (the quantum of conductance), small plateaus in $G$ followed by a sharp drop indicate the final breakdown of the metallic junction. Below this, and following the initial jump-out-of-contact (JOC), we observe exponential tunneling if no molecule is wired or, in the case a molecule is wired, conductance plateaus develop which stand out from the exponential background. We recorded thousands of opening/closing cycles per experiment, and we repeated each measurement with fresh electrodes to test for potential variation in the gold breaking dynamics. In all experiments we use a tip-sample bias of $0.23 \mathrm{~V}$.

To process the data, we run an algorithm that separates molecular junctions from tunneling-only junctions, and from this we create $2 \mathrm{D}$ density plots (histograms) of the molecular conductance at all electrode separations. To calculate the plateau lengths for each individual $G_{z}$ trace, we measure the distance between two points in each trace, the first just after the $\mathrm{Au}-\mathrm{Au}$ junction cleavage $\left(\log \left(G / G_{0}\right)\right.$ $=-0.5$ ) and the other being the last point in the trace which occurs 1 order of magnitude below the main conductance (determined from the $1 \mathrm{D}$ histogram peak). Finally, we add $0.4 \mathrm{~nm}$ to account for the initial jump-out-of-contact (see SI Figure S26 for further details). This is the minimum jump we expect to occur and means we do not overestimate the calibrated distance. Each individual length is then plotted in a histogram to show the total distribution.

\section{RESULTS AND DISCUSSION}

First, we separate the plateau-containing traces from the tunneling-only traces, which allows us to determine the percentage of total junctions displaying molecular junction formation. These are shown in Figure 2, and the tunneling-only traces can be found in Figure S21. For all S2 compounds (SAc and $\mathrm{SMe}$ ) we find this percentage falls between 7-14\%, with 
no obvious bias toward one termination or isomer. These rates are generally lower than typically found for oligo(phenylene ethynylene) (OPE)-based compounds measured under similarly solvent-free conditions. For either OPE3-diamines or methyl sulfides (where 3 describes the number of phenyl groups), plateaus are found in generally $25-60 \%$ of all traces. For OPE3-dithiols, this drops to about $12-26 \%{ }^{33,34} \mathrm{We}$ conclude that junction formation for the porphyrin compounds is to a certain extent influenced by the porphyrin unit itself. This is understandable given that porphyrins are well-known to bind strongly to metal surfaces, ${ }^{35}$ and the lower percentages of junctions are consistent with a reduced surface mobility compared to OPEs.

As can be seen in Figure 2 for the majority of S2 compounds, two groups of plateaus can be seen, an upper group (labeled G1) in which the plateaus are generally shorter, and a lower group (G2) with correspondingly longer plateaus. Using our analysis algorithm, we have further subdivided these traces depending on whether they contain a G1 or a G2 plateau, or both (Figure 3). We have collated the plateau percentage data in Table S2. We find that G1 and G2 plateaus can occur either independently or together within the same trace, and we find that G2 plateaus are generally the most probable, regardless of isomer or binding group. Another significant finding is that traces containing both a G1 and a G2 plateau occur more frequently for the trans isomers than for the cis, both for SAc and SMe. This will be discussed later in the text. It is also remarkable that the cis compounds can bind at all to the electrodes, given the position of the sulfur atoms. We note, however, that the conformational flexibility of porphyrins is well-known, ${ }^{36}$ and a $10-15^{\circ}$ out-of-plane distortion could more easily allow the sulfurs to bind to gold, as long as the electrodes are quite sharp, as is the case in BJs.

The conductance for each group is given in Table 1 , where we have fitted a Gaussian to the peak of the $1 \mathrm{D}$ histogram

Table 1. Mean 1D Log-Histogram Peak Maxima for Each Compound Measured over Two Separate Runs ${ }^{a}$

$\begin{array}{lccc} & G_{\mathrm{G} 1}\left(\log \left(G / G_{0}\right)\right) & G_{\mathrm{G} 2}\left(\log \left(G / G_{0}\right)\right) & G_{\mathrm{G} 2} *\left(\log \left(G / G_{0}\right)\right) \\ \text { S2-trans-SMe } & -3.2 & -4.6 & -4.8 \\ \text { S2-cis-SMe } & -3.0 & -4.6 & -4.8 \\ \text { S2-trans-SAc } & -2.6 & -4.1 & -4.4 \\ \text { S2-cis-SAc } & -2.5 & -4.1 & -4.4\end{array}$

${ }^{a} G_{\mathrm{G} 2} *$ is the value at maximum junction extension (see SI Figures S24 and 25).

generated from the G1/G2-only traces, and here we quote the maximum value. There is a clear difference in the conductance for both G1 and G2 between SAc termination and the SMe equivalents, with the SAc values approximately a factor of 4 higher than SMe (for the 1D histograms we refer to Figure S22). We find, however, no discernible differences in the conductance between cis and trans isomers with the same binding group. We note, however, that G1 for both S2-cis-SMe and S2-trans-SMe is more weakly defined than the SAc analogues.

The plateau lengths for each group are given in Tables S3 (G1) and S4 (G2). The distance between the last gold atoms of each pyramidal electrode attached to the sulfur groups (as generated by our DTF calculations) is $1.5 / 1.6 \mathrm{~nm}$ for S2-transSAc/SMe and 1.2/1.3 nm for S2-cis-SAc/SMe. On the basis of this, we expect plateaus for the trans isomers to be, on average, about $0.3 \mathrm{~nm}$ longer than for cis isomers. The experimentally measured plateau lengths can be fitted quite well with a single Gaussian distribution, from which we can define a mostprobable stretching value (the peak maximum, $L_{\mathrm{p}}$ ) and a maximum stretching value (the 95 th percentile, $L_{95}$ ). The maximum stretching value may be related to junctions in which the molecule is either pulled along the electrodes the furthest to a smaller JOC or to the extrusion of gold atoms. We have checked the longest plateaus and see a tendency for the JOC to be less for these junctions. There is still, however, an appreciable initial retraction of about $0.4 \mathrm{~nm}$ (see Figure S26 in SI for details of the JOC calibration). Generally, we find the JOC varies between 0.4 to $0.6 \mathrm{~nm}$ over the entire measurement, and as it is impossible to calibrate each individual junction separately, we simply calibrate all junctions using the lowerdetermined distance. This means we will underestimate the real electrode separation in some junctions, but importantly we do not overestimate the longest junctions. Finally, we quote all distances as the mean over two separate experimental runs.

Looking at the SMe-terminated isomers first, we determined $L_{\mathrm{p}}\left(L_{95}\right)$ for S2-trans-SMe to be $0.90(1.18) \mathrm{nm}$ for G1 and $1.10(1.42) \mathrm{nm}$ for G2. For S2-cis-SMe, these values were 0.76 (0.99) $\mathrm{nm}$ for G1 and $0.92(1.22) \mathrm{nm}$ for G2. It is clear that for both isomers the $L_{95}$ values of G2 correspond very well with the expected maximum $\mathrm{Au}-\mathrm{Au}$ separations for a single molecule junction. We also find the difference in plateau length between cis and trans isomers (the difference in $L_{\mathrm{p}}\left(L_{95}\right)$ is $0.18(0.20)$ $\mathrm{nm}$ ), which is close to the anticipated value of $0.3 \mathrm{~nm}$. This allows us to associate with confidence G2 plateaus to junctions in which transport takes place between the two sulfur atoms bound to each electrode. This behavior is akin to that seen for the family of alkanediamines studied by Arroyo et al., where the longest plateaus did not exceed the calculated junction length. ${ }^{37}$

Turning to the SAc-terminated isomers next, in which the acetyl group is known to be cleaved upon exposure to gold under the conditions used here, ${ }^{38}$ we determined $L_{\mathrm{p}}\left(L_{95}\right)$ for S2-trans-SAc to be $0.95(1.38) \mathrm{nm}$ for G1 and $1.40(2.02) \mathrm{nm}$ for G2. For S2-cis-SAc, these values were $1.02(1.41) \mathrm{nm}$ for G1 and 1.47 (2.02) nm for G2. These values are surprising for several reasons. First, we see that there is hardly any difference in plateau length distributions between cis and trans isomers. Second, the $L_{95}$ values greatly exceed the calculated maximum lengths by $0.5 \mathrm{~nm}$ in the case of the trans isomer and by $0.8 \mathrm{~nm}$ in the case of the cis isomer. Even the $L_{\mathrm{p}}$ value of G2 for the cis isomer is greater than the $1.2 \mathrm{~nm}$ calculated junction length, and so there is clearly a different junction-breaking mechanism at work during junction evolution of the thiolates, again in agreement with the findings of Arroyo et al. ${ }^{37}$

Figure $2 \mathrm{E}$ shows the $2 \mathrm{D}$ histogram of a mono-SAc (S1) variant, which cannot form sulfur-to-sulfur bound junctions. As we only observe one conductance group in this case, which occurs at the same conductance as G1 for the S2 variants, this proves that the current in these junctions must follow a path other than the $S-S$ pathway. For the $S 1$, the calibrated maximum plateau length $\left(L_{95}\right)$ corresponds well to the calculated distance between a gold atom attached to the sulfur and the opposing edge of the porphyrin ring (both $1.1 \mathrm{~nm}$ ). Taking into account the short nature of these plateaus, we conclude that G1 involves a direct coupling between the porphyrin and one of the electrodes, in effect bypassing one of the sulfur contacts in the S2 compounds. This might include either the central zinc ion, the $\pi$-system, or a combination of both. This distance is slightly shorter than measured for G1 for 
the S2 SAc compounds, which may be due to the presence of two $S$ atoms in the $S 2$ case. The $S 1$ plateau lengths are about the same as we find for the S2 SMe compounds, although it must be stressed that, especially for S2-cis-SMe, G1 is less welldefined, which may be a result of the weaker interaction with gold by the SMe group. It is interesting to note that the ratio of conductance between $\mathrm{G} 1_{\mathrm{SMe}}$ and $\mathrm{G} 1_{\mathrm{SAc}}$ is the same as with $\mathrm{G} 2_{\mathrm{SMe}}$ and $\mathrm{G} 2_{\mathrm{SAc}}$ indicating that the anchor groups still play a role in determining the overall conductance despite not fully contributing to the current pathway in G1.

The observation of a separate conductance group corresponding to a $\mathrm{Au}$-porphyrin direct interaction independently corroborates the low overall percentage of plateaus for both SAc and SMe compounds. This interpretation also allows us to understand the observation that for both cis isomers (SMe and SAc) we consistently find fewer junctions with a transition from G1 to G2 compared to the trans counterparts. The mean percentage of these traces for the trans-SMe isomer (over two runs) is $7.7 \%$, which compares to just $2.4 \%$ for the cis-SMe isomer (see Table S2 for a breakdown of each run). For the trans-SAc isomer, we find $19.9 \%$ compared to only $9.0 \%$ for the cis-SAc isomer. Our explanation for this is the following. In the case of the cis isomers, if the central porphyrin ring binds to one electrode, with one $S$ group attached to the other, the second $S$ group cannot easily attach to the same electrode as the porphyrin ring. This means that once the $\mathrm{Au}$-porphyrin contact breaks due to stretching, the whole molecule must reorient itself in order for the second $S$ group to bind. The scenario is not the same for the trans isomers, where the natural orientation of the molecule in the junction favors attachment of both $\mathrm{S}$ groups, even when there is a direct $\mathrm{Au}$-porphyrin interaction. We have attempted to represent these scenarios in Figure S23.

We now return to understanding the mechanism underlying the qualitatively different behavior of G2 for both cis and trans SAc compounds compared with the SMe counterparts. The plateau length distributions $(L)$ for G2 are shown in Figure 4.

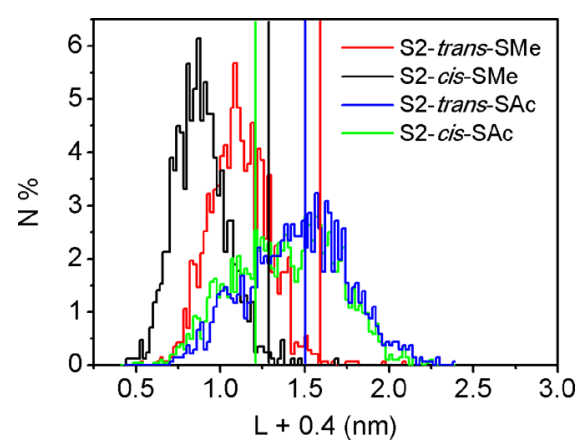

Figure 4. Calibrated G2 plateau length distributions for the average of two runs per compound. $L$ denotes the displacement length as recorded in the measurement. We add $0.4 \mathrm{~nm}$ to account for the JOC. Vertical lines represent the calculated junction lengths between the final $\mathrm{Au}$ atoms.

We begin by noting that OPE3-dithiols generally show measurably longer plateaus than the methylsulfide- and amine-terminated analogues, by 0.2 to $0.3 \mathrm{~nm}$, despite having practically the same molecular length. ${ }^{6}$ This value is in reasonable agreement with the difference in $L_{\mathrm{p}}$ and $L_{95}$ we find between G2 of S2-trans-SMe and S2-trans-SAc (0.3 and $0.5 \mathrm{~nm}$, respectively). We can understand this in terms of the stronger $\mathrm{Au}-\mathrm{S}$ thiolate bond compared with the weaker dative interaction of the SMe. This implies that, under tension, the electrodes will deform elastically by an amount similar to that of a $\mathrm{Au}-\mathrm{Au}$ atomic junction $(0.2-0.4 \mathrm{~nm})$. Conversely, S2-cisSAc shows significantly longer plateaus than S2-cis-SMe, with $L_{\mathrm{p}}$ and $L_{95}$ values greater by 0.5 and $0.8 \mathrm{~nm}$, respectively. These differences are hard to explain by just considering deformation of the contacts at room temperature. The extra length can, however, be accounted for satisfactorily if we assume that the cis isomer undergoes isomerization to the trans isomer during elongation of the junction. As this would be a mechanically induced effect, S2-trans-SAc could not be converted to S2-cisSAc. The overlap in the plateau length distributions of the two isomers would suggest that this happens in most junctions; otherwise a greater proportion of shorter plateaus would be present, which we do not find. In the following we provide further evidence for atropisomerization using a kinetic and theoretical analysis.

A kinetic analysis of the cis/trans atropisomerization equilibrium in solution (Figure 5) reveals that the rotational

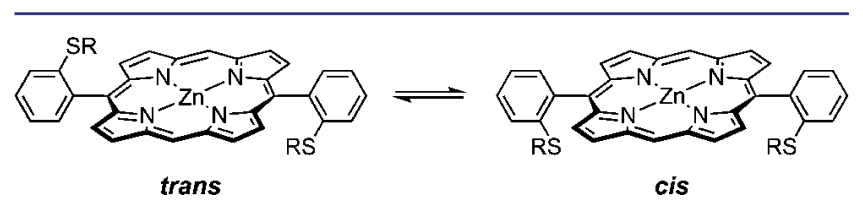

Figure 5. Atropisomerization equilibrium between cis and trans compounds $(\mathrm{R}=\mathrm{Ac}, \mathrm{Me})$. The isomers are stable in solution at room temperature but exchange upon heating or due to strain in a molecular junction.

barriers around the porphyrin-aryl bond in SAc and SMe compounds are very similar (see also SI pages S18-S20 for further details). Neither the SAc nor the SMe sets of compounds isomerize at room temperature; however, at 140 ${ }^{\circ} \mathrm{C}$ in 1,1,2,2-tetrachloroethane- $d_{2}$ their half-lives drop to 15 and $11 \mathrm{~h}$, respectively (see SI for details). Under these conditions, we measure free energies of activation for the rotation around a porphyrin-aryl bond of $\Delta G^{\ddagger}=145.8( \pm 0.4)$ $\mathrm{kJ} \mathrm{mol}^{-1}$ and $144.7( \pm 0.6) \mathrm{kJ} \mathrm{mol}^{-1}$ for the SAc and SMe compounds, respectively. The rotational barriers are higher than reported values for zinc porphyrins with ester, ${ }^{39}$ amide, ${ }^{40}$ or cyano ${ }^{41}$ ortho substituents, which is consistent with the larger size of the sulfur atom compared to oxygen, nitrogen, and carbon atoms. Our results are also in agreement with molecular models showing that the barrier to rotation originates mostly from the bulk of the sulfur atom rather than its methyl or acetyl substituents. They suggest that the difference in molecular junction behavior between S2-cis-SAc and S2-cis-SMe is due to the difference in bond strength between the molecule and the gold electrodes: for S2-cis-SMe, the molecular junction breaks before isomerization occurs, while for S2-cis-SAc, the S-Au bond is strong enough to enable a strain-induced isomerization in the molecular junction. We used the Eyring equation to estimate the enthalpy and entropy of activation from the rates of atropisomerism at $120{ }^{\circ} \mathrm{C}$ and $140{ }^{\circ} \mathrm{C}$. This analysis gives $\Delta H^{\ddagger}=85( \pm 15) \mathrm{kJ} \mathrm{mol}^{-1}$ and $\Delta S^{\ddagger}=-146( \pm 38) \mathrm{J} \mathrm{mol}^{-1} \mathrm{~K}^{-1}$ for SAc and $\Delta H^{\ddagger}=88( \pm 14) \mathrm{kJ} \mathrm{mol}^{-1}$ and $\Delta S^{\ddagger}=-138( \pm 36)$ $\mathrm{J} \mathrm{mol}^{-1} \mathrm{~K}^{-1}$ for $\mathrm{SMe}$, in $\mathrm{C}_{2} \mathrm{Cl}_{4} \mathrm{D}_{2}$. Changing the solvent to DMSO- $d_{6}$ gave faster rates of isomerization, although kinetic experiments were less reproducible in this solvent, possibly due to competing decomposition. 

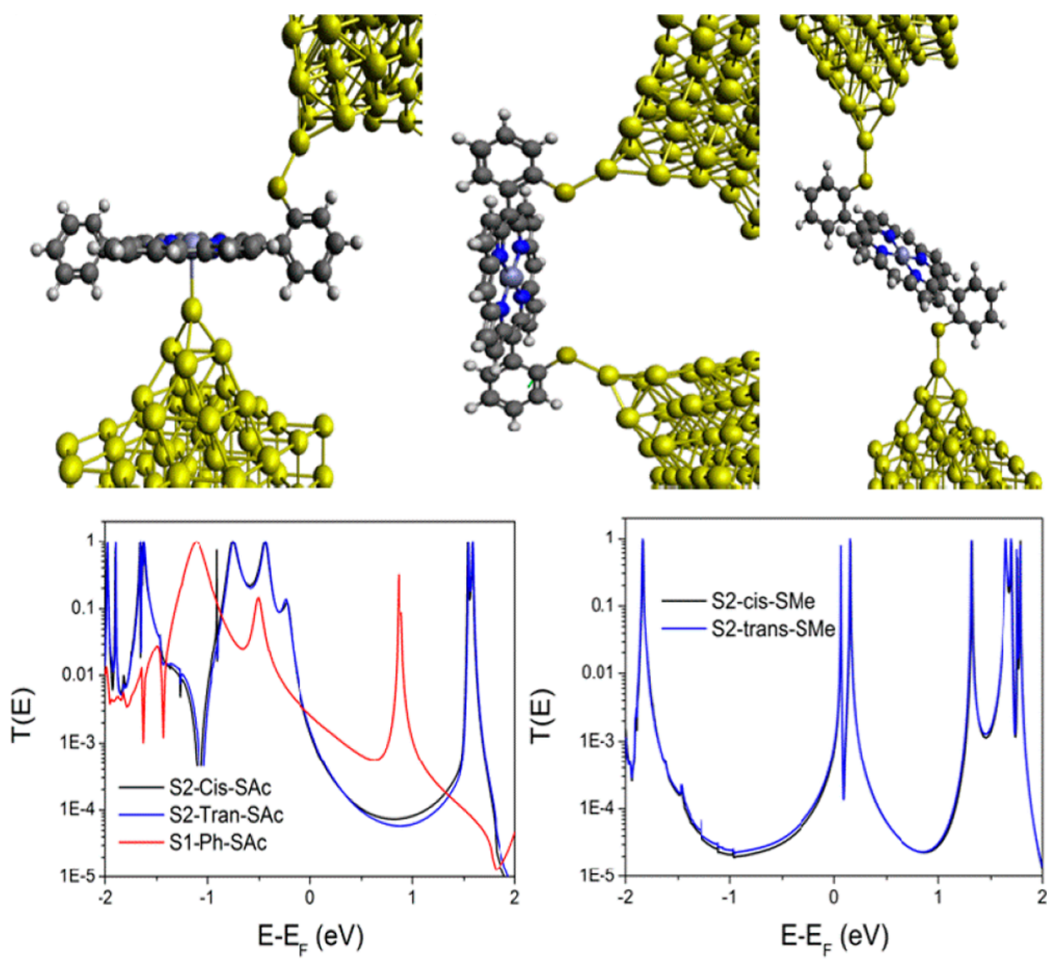

Figure 6. (Top) Contact geometries for the SAc series of molecules (left) G1, G2-cis (middle), and G2-trans (right). (Bottom) Zero-bias transmission coefficient $T(E)$ vs electron energy for SAc anchor group (left) and SMe anchor group (right).

Using these values, we can estimate the barrier at room temperature to compare with the BJ experiments, which we find $\Delta G^{\neq}=129( \pm 19) \mathrm{kJ} \mathrm{mol}^{-1}(1.34 \mathrm{eV})$ for SAc, and the same value $129( \pm 18) \mathrm{kJ} \mathrm{mol}^{-1}(1.29 \mathrm{eV})$ for SMe. Okuno et al. calculated the reaction path and potential energy barrier for phenyl ring rotation in $o$-methoxyphenylporphyrin, which is a similar structure to our $\mathrm{SMe} / \mathrm{SAc}$ compounds. They found a barrier of $105 \mathrm{~kJ} \mathrm{~mol}^{-1}(1.09 \mathrm{eV})$, which is comparable to the values we have measured. ${ }^{42}$

\section{THEORETICAL MODELING}

To explain the conductance measurements, we perform ab initio-based quantum transport calculation using the Gollum code. ${ }^{43}$ First, the molecules in Figure 1 were relaxed to their optimum geometries using the density functional theory code SIESTA. ${ }^{44}$ A double- $\zeta$ polarized basis set was utilized along with norm-conserving pseudopotentials, and the exchange correlation functional was described by the generalized gradient approximation. $^{45}$ The fineness of the real space grid was defined by a mesh cutoff of $150 \mathrm{Ry}$, and a force tolerance of $0.01 \mathrm{eV} / \AA$ was used to determine the ground-state geometry. The aryl rings have a torsion angle of $69^{\circ}$ for the SAc and SMe anchor groups and $63^{\circ}$ for the nonfunctionalized ring, in keeping with a statistical analysis of conformations in crystal structures. ${ }^{46}$ The difference in the ground state energy for S2trans-SAc and S2-cis-SAc is $0.005 \mathrm{eV}(0.003 \mathrm{eV}$ for SMe).

To calculate the electronic conductance, we attach the molecules to gold electrodes, which consist of six layers of (111) gold each containing 25 atoms and a pyramid of 11 gold atoms and extract a Hamiltonian describing this extended molecule using SIESTA. For the SAc anchor group, the optimum binding geometry to the gold tip via the terminal sulfur atom is calculated to be a distance of $2.45 \AA$ and a $\mathrm{Au}-$ $\mathrm{S}-\mathrm{C}$ angle of $120^{\circ}$ with a binding energy (BE) of $1.6 \mathrm{eV}$. The
$\mathrm{BE}$ agrees well with similar molecules calculated elsewhere. ${ }^{47}$ For SMe anchor groups, the binding distance is slightly larger at $2.55 \AA$ and the angle is $117^{\circ}$ with a much smaller BE of $0.35 \mathrm{eV}$. The binding for the G1 conductance group where one gold tip is attached to the core porphyrin unit, the optimum location is found to be directly above the central zinc atom, with a binding distance of $2.9 \AA$ and a BE of $0.15 \mathrm{eV}$. The zero-bias transmission coefficient $T(E)$ for each of these contact geometries obtained using the Gollum code are shown in Figure 6.

The transmission data show that the curves for the S2-cisSAc and S2-trans-SAc the $T(E)$ curves are almost identical, with the DFT-predicted Fermi energy $E_{\mathrm{F}}$ sitting close to the HOMO resonance of the molecule, which is typical behavior for thiol anchor groups. The value of $\log \left(G / G_{0}\right)=\log T\left(E_{\mathrm{F}}\right)$ is -2.8 for cis and -2.9 for trans. For S1-Ph-SAc, the conductance is higher with a value of -2.4 . These values are greater than the experimental measurements in Table 1 . The overestimation arises due to known problems with DFT in predicting the correct position of the Fermi energy. ${ }^{48}$ In practice, $E_{\mathrm{F}}$ usually lies closer to the middle of the HOMO-LUMO gap, which in our case is approximately $E-E_{\mathrm{F}}=0.5 \mathrm{eV}$. From the value of $T(E)$ at this energy, we find that $\log G / G_{0}$ is -3.98 for S2-cisSAc, -4.02 for S2-trans-SAc, and -3.2 for S1-Ph-SAc, which is in much better agreement with experiment. The calculated gold-gold separation for each of these three junctions is 1.5 $\mathrm{nm}$ (S2-trans-SAc), $1.2 \mathrm{~nm}$ (S2-cis-SAc), and $0.96 \mathrm{~nm}$ (S1-Ph$\mathrm{SAc})$.

The transmission curves for the SMe-terminated molecules show similar behavior (Figure 6) with the cis and trans configurations possessing almost identical transmission curves. Again the gold-gold separations are different ( $1.3 \mathrm{~nm}$ cis, 1.6 nm trans). The DFT-predicted Fermi energy now lies close to the LUMO resonance, which would lead to conductances 
which are too high. Shifting this toward the middle of the gap to an energy $E-E_{\mathrm{F}}=-0.5 \mathrm{eV}$ yields conductance values $\log \left(G / G_{0}\right)$ of -4.43 for S2-cis-SMe and -4.50 for S2-transSMe, which are lower than the conductance values for the thiol series in agreement with the measured values in Table 1.

The energy barrier for rotation of the end aryl group about the porphyrin axis is large due to the steric effects of the additional thiol and SMe groups in the ortho position. A detailed description of the energy landscape of these molecules is given in SI (section 3). If the porphyrin is constrained to retain its optimum geometry, the energy barrier is extremely high. However, if the porphyrin is allowed to deform within the junctions, then the energy barrier is significantly lower. ${ }^{42}$ Also, the $\mathrm{BE}$ of the gold-gold bond at the apex of a pyramid of gold atoms is calculated to be $1.62 \mathrm{eV}$, which is slightly larger than that of the gold thiol bond. For thiols experimentally, the mean breakdown force of individual junctions has been measured as close to $1.5 \mathrm{nN}^{49,50}$ (the same as that for a $\mathrm{Au}-\mathrm{Au}$ bond, implying breakdown of thiolated junctions could occur either between $\mathrm{Au}-\mathrm{Au}$ or $\mathrm{Au}-\mathrm{S}$ bonds). For $\mathrm{SMe}$, the junction breakdown force has been measured to be $0.5 \mathrm{nN},{ }^{51}$ For cis-totrans conversion to be induced by the junction, the amount of energy needed to break the bond at the contact must be larger than the barrier to atropisomerization. On the basis of the $\mathrm{BE}$ calculations, which give a larger $\mathrm{BE}$ for the thiol than the measured rotational barrier, but a lower $\mathrm{BE}$ in the case of SMe, as well as the experimental force measurements, it seems perfectly plausible that during the stretching of thiol junctions, more work is required to break the $\mathrm{Au}-\mathrm{Au} / \mathrm{Au}-\mathrm{S}$ bond than to overcome the rotational barrier; hence, isomerization can take place. This clearly is not the case for SMe, where the junction breaks before isomerization can occur (this is represented schematically in Figure 7).

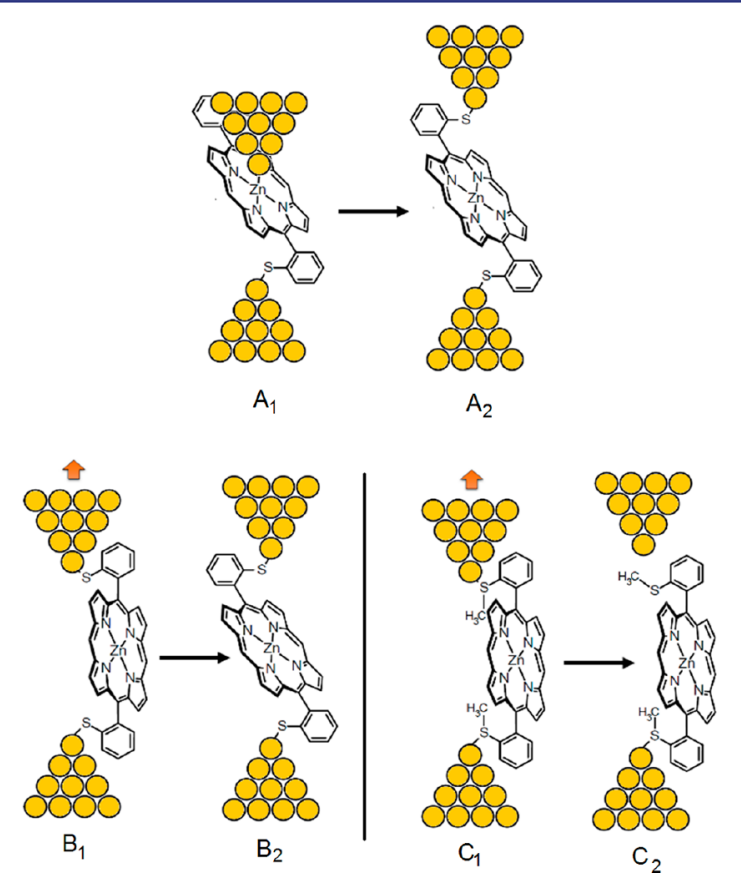

Figure 7. Different configurations and transitions of the molecules between the electrodes. $A_{1}$ to $A_{2}$ represents the transition from group 1 (G1) to group 2 (G2). B1 to B2 represents atropisomerization of S2cis-SAc to S2-trans-SAc. C1 to C2 describes the SMe-anchored cis junction, which cleaves before isomerization can occur.
We have carried out a theoretical analysis of a possible transition state geometry using a representative isolated molecule. For details of the methods used, see SI section 5 . This was carried out by rotating one aryl group stepwise and by evaluating the energy of the system at each step. For each point, freezing some atoms was necessary in order to prevent the system from regaining the original structure. By performing such an analysis, a barrier of $1.77 \mathrm{eV}$ was obtained, and we show the transition structure (at $\alpha \approx 145^{\circ}$ ) in Figure S32 (lower right panel). This possible energy pathway is in good agreement with the experimentally determined value, although we are still overestimating the value somewhat due to the restrictions imposed.

This mechanism indicates that the porphyrin adopts significantly nonplanar conformations inside the junction, which is something seldom considered, especially for conjugated $\pi$-systems. Such deviations from planarity may be important when trying to understand the conductance of long organic molecules and also flexible biomolecules such as proteins and DNA. Due to the size of the barrier, we do not expect thermal fluctuations or bias voltage $(0.23 \mathrm{~V}$ used here) to play a significant role in the isomerization process. The observed relation between isomerization and moleculeelectrode binding group strongly implies that this is a mechanically driven phenomenon. Although an exact value for the barrier cannot be extracted from the experiments, we can place upper and lower limits of 1.6 and $0.35 \mathrm{eV}$ using the $\mathrm{BE}$ of $\mathrm{Au}-\mathrm{S}$ and $\mathrm{Au}-\mathrm{SMe}$ groups, respectively. This fits well with the value from our kinetic analysis of $1.34 \mathrm{eV}$ (S2-transSAc). To obtain greater precision would require studying other binding groups, with a greater range of $\mathrm{BE}$.

We do not observe an obvious transition in any of the individual traces or the $2 \mathrm{D}$ histogram for the isomerization of S2-cis-SAc. This could be for several reasons. First, the isomerization most likely occurs on time scales much too short to be detected by our instrumentation (molecular motions are generally on the picosecond scale). This would explain why the conductance we observe for cis/trans isomers is the same (within error). Despite the implication that the torsion angle between one of the aryl groups and the central porphyrin ring of S2-cis-SAc varies more during stretching compared to S2-trans-SAc, which would be expected to produce a change in conductance, ${ }^{52}$ the likely quick nature of this process is not expected to produce a noticeable change in the average distribution of torsion angles across the junction. Also, individual traces fluctuate by about 1 order of magnitude in conductance due to changes in molecule-electrode contact, and this may mask traces of the isomerization process. ${ }^{33} \mathrm{We}$ notice too that all wired cis-thiol molecules seem to be isomerized, which we infer due to the absence of a higher proportion of shorter plateaus for S2-cis-SAc compared to the trans.

Another interesting, and perhaps surprising, outcome is that the cis configuration can bind to the electrodes at all. Steric hindrance between the $\mathrm{H}$ atoms of the phenyl ring and gold might be expected to strongly reduce the possibility of wiring. We note, however, that the flexibility of the porphyrin, especially in the presence of gold as we have shown, can quite conceivably lead to more favorable conformations that allow $\mathrm{Au}-\mathrm{S}$ binding at both ends of the molecule. This highlights once again that understanding conformational degrees of freedom inside molecular junctions is key for a sound interpretation of results. 
The method we have outlined for controlling molecular conformation using different anchor groups could be applied to any system with one, or several, internal conformational barriers. We envisage that this could be used, perhaps, to shed more light on the effect of unraveling of $\mathrm{H}$-bonded molecules or the unfolding of DNA, peptides, or proteins on their transport properties. Having weaker binding groups would allow molecular conformations to persist under stretching, whereas stronger binding groups would force the molecule to unravel, allowing for a detailed comparison of both situations.

We further point out that knowledge of the anchor group binding strength and internal atropisomerization barriers can be considered complementary, and in principle the variation of one could be used to elucidate the other to a reasonable degree of accuracy. This might lead to more elaborate studies in which anchor group binding strengths can be assessed through internal reference barriers, complimenting the force measurements currently performed. ${ }^{50,53}$

\section{CONCLUSIONS}

We have studied a family of porphyrin compounds in which the binding groups (either SAc or SMe) are located ortho to the central porphyrin on the outer phenyl rings, giving rise to two distinct atropisomers, cis and trans. For all S2 compounds, two conductance groups were observed (an upper G1 and a lower G2), the conductance values of which do not depend significantly on the isomerization of the molecule. The SMe anchor gave conductance values consistently lower than that of SAc, by about a factor of 4. Replacing one of the terminal $\mathrm{PhSAc}$ groups with a $\mathrm{Ph}$ group inhibited the lower $\mathrm{G} 2$ plateaus but still displayed the upper G1, showing that this group is related to a shorter conductance path which does not involve both sulfur atoms. The plateau lengths suggest binding to the central porphyrin unit. Our theoretical study fully supports these observations, and we can reproduce G1 and G2 using the experimentally inferred binding locations. We also show theoretically that the conductance does not depend on the trans or cis configuration even though the electrode separations differ.

For the S-S pathway junctions (G2), we found consistent differences in the breakdown distance distributions for the $\mathrm{SMe}$ compounds, the cis isomer showing plateaus overall shorter than that of the trans isomer. We found the maximum observed breakdown distance fits well to the calculated junction length for each compound, and practically no junctions exceed this value. In contrast, the SAc compounds showed no noticeable difference in breakdown distance, and both often exceed the calculated fully stretched value, which for the cis isomer was up to $0.8 \mathrm{~nm}$ beyond this value. In order to reconcile this, deformation of the contacts explains part of the "extra" distance, but an additional mechanism must be at work. We investigated the potential energy barrier to ring rotation for the outer phenyl groups, carrying out a kinetic analysis of the cis/ trans atropisomerization equilibrium in solution. From this we estimated the room temperature barrier to be $129 \mathrm{~kJ} \mathrm{~mol}^{-1}$ $(1.34 \mathrm{eV})$ for both SAc and SMe. Our theoretical calculations showed that the $\mathrm{Au}-\mathrm{S}$ (for thiolate connection) and $\mathrm{Au}-\mathrm{Au}$ bond energies are greater than this $(1.60$ and $1.62 \mathrm{eV}$, respectively), whereas the $\mathrm{Au}-\mathrm{SMe}$ bond is significantly weaker $(0.35 \mathrm{eV})$. We infer, therefore, that for the cis-SAc isomer, strain-induced cis-to-trans isomerization takes place during the stretching of a single molecule junction. This process does not occur for the SMe analogue. For isomerization to occur, the porphyrin must adopt significantly nonplanar conformations within the junction, which stresses the importance of considering molecular deformation inside junctions.

\section{ASSOCIATED CONTENT}

\section{Supporting Information}

The Supporting Information is available free of charge on the ACS Publications website at DOI: 10.1021 jacs.7b10542.

All synthetic procedures and analysis, details of the kinetic study of atropisomerization, X-ray crystal structures, general methods for the BJ study plus further analysis, theoretical binding energy and ground-state energy analyses (PDF)

X-ray data for S2-trans-SMe (CIF)

\section{AUTHOR INFORMATION}

\section{Corresponding Authors \\ *E.Leary@liverpool.ac.uk \\ *harry.anderson@chem.ox.ac.uk \\ *c.lambert@lancaster.ac.uk \\ *nichols@liverpool.ac.uk \\ ORCID}

Edmund Leary: 0000-0001-7541-5997

Carlos Romero-Muñiz: 0000-0001-6902-1553

Yaoyao Xiong: 0000-0002-3741-7454

Maria A. Lebedeva: 0000-0002-3543-6416

Colin J. Lambert: 0000-0003-2332-9610

Harry L. Anderson: 0000-0002-1801-8132

Richard J. Nichols: 0000-0002-1446-8275

\section{Author Contributions}

The manuscript was written through contributions of all authors. All authors have given approval to the final version of the manuscript.

\section{Notes}

The authors declare no competing financial interest.

\section{ACKNOWLEDGMENTS}

R.J.N., S.H., E.L., and A.H. acknowledge the UK Engineering and Physical Sciences Research Council (EPSRC) for the funding of this research under grant numbers EP/M014169/1, P/M029522/1, and EP/M005046/1. N.A., C.L., I.G., and Q.A.G. acknowledge the European Commission (EC) FP7 ITN "MOLESCO" (project no. 606728) and the EPSRC (grant nos. EP/M014452/1 and EP/N017188/1). H.L.A., C.R., and H.W.J. thank the EPSRC (grant EP/M016110/1), UK National Mass Spectrometry Facility at Swansea University, for recording mass spectra and Dr. Amber L. Thompson for help with X-ray crystal structure refinements. K.P. and M.L. acknowledge the EPSRC grant EP/K030108/1. N.A. and G.R.B. acknowledge the Spanish MINECO (Grant MAT2014-57915-R). IMDEA Nanociencia acknowledges support from the 'Severo Ochoa' Programme for Centres of Excellence in R\&D (MINECO, Grant SEV-2016-0686). We thank Linda A. Zotti for useful discussion.

\section{REFERENCES}

(1) Su, T. A.; Neupane, M.; Steigerwald, M. L.; Venkataraman, L.; Nuckolls, C. Nat. Rev. Mater. 2016, 1, 16002.

(2) Van Dyck, C.; Ratner, M. A. J. Phys. Chem. C 2017, 121, 3013.

(3) Perrin, M. L.; Burzurí, E.; van der Zant, H. S. Chem. Soc. Rev. 2015, 44, 902. 
(4) Cuevas, J. C.; Scheer, E. Molecular electronics: An introduction to theory and experiment; World Scientific: Hackensack, NJ, 2010.

(5) Quek, S. Y.; Kamenetska, M.; Steigerwald, M. L.; Choi, H. J.; Louie, S. G.; Hybertsen, M. S.; Neaton, J. B.; Venkataraman, L. Nat. Nanotechnol. 2009, 4, 230.

(6) Miguel, D.; Alvarez de Cienfuegos, L.; Martín-Lasanta, A.; Morcillo, S. P.; Zotti, L. A.; Leary, E.; Bürkle, M.; Asai, Y.; Jurado, R.; Cárdenas, D. J.; et al. J. Am. Chem. Soc. 2015, 137, 13818.

(7) Wang, L.; Gong, Z.; Li, S.; Hong, W.; Zhong, Y.; Wang, D.; Wan, L. Angew. Chem. 2016, 128, 12581.

(8) Bruot, C.; Xiang, L.; Palma, J. L.; Li, Y.; Tao, N. J. Am. Chem. Soc. 2015, 137, 13933.

(9) Li, L.-L.; Diau, E. W.-G. Chem. Soc. Rev. 2013, 42, 291.

(10) Mathew, S.; Yella, A.; Gao, P.; Humphry-Baker, R.; Curchod, B. F.; Ashari-Astani, N.; Tavernelli, I.; Rothlisberger, U.; Nazeeruddin, M. K.; Grätzel, M. Nat. Chem. 2014, 6, 242.

(11) Armaroli, N.; Accorsi, G.; Song, F.; Palkar, A.; Echegoyen, L.; Bonifazi, D.; Diederich, F. ChemPhysChem 2005, 6, 732.

(12) Xu, B.; Tao, N. Science 2003, 301, 1221.

(13) Nichols, R. J.; Haiss, W.; Higgins, S. J.; Leary, E.; Martin, S.; Bethell, D. Phys. Chem. Chem. Phys. 2010, 12, 2801.

(14) Bennett, N.; Xu, G.; Esdaile, L. J.; Anderson, H. L.; Macdonald,

J. E.; Elliott, M. Small 2010, 6, 2604.

(15) Sedghi, G.; García-Suárez, V. M.; Esdaile, L. J.; Anderson, H. L.; Lambert, C. J.; Martín, S.; Bethell, D.; Higgins, S. J.; Elliott, M.; Bennett, N.; et al. Nat. Nanotechnol. 2011, 6, 517.

(16) Liu, Z.-F.; Wei, S.; Yoon, H.; Adak, O.; Ponce, I.; Jiang, Y.; Jang, W.-D.; Campos, L. M.; Venkataraman, L.; Neaton, J. B. Nano Lett. 2014, 14, 5365.

(17) Li, Z.; Park, T.-H.; Rawson, J.; Therien, M. J.; Borguet, E. Nano Lett. 2012, 12, 2722.

(18) Noori, M.; Aragonès, A. C.; Di Palma, G.; Darwish, N.; Bailey, S. W. D.; Al-Galiby, Q.; Grace, I.; Amabilino, D. B.; González-Campo, A.; Díez-Pérez, I.; Lambert, C. J. Sci. Rep. 2016, 6, 37352.

(19) Chen, F.; Hihath, J.; Huang, Z.; Li, X.; Tao, N. Annu. Rev. Phys. Chem. 2007, 58, 535.

(20) Widawsky, J.; Kamenetska, M.; Klare, J.; Nuckolls, C.; Steigerwald, M.; Hybertsen, M.; Venkataraman, L. Nanotechnology 2009, 20, 434009.

(21) Reddy, P.; Jang, S.-Y.; Segalman, R. A.; Majumdar, A. Science 2007, 315, 1568.

(22) Evangeli, C.; Gillemot, K.; Leary, E.; González, M. T.; RubioBollinger, G.; Lambert, C. J.; Agraï, N. Nano Lett. 2013, 13, 2141.

(23) Sedghi, G.; Sawada, K.; Esdaile, L. J.; Hoffmann, M.; Anderson, H. L.; Bethell, D.; Haiss, W.; Higgins, S. J.; Nichols, R. J. J. Am. Chem. Soc. 2008, 130, 8582.

(24) Li, Z.; Smeu, M.; Park, T.-H.; Rawson, J.; Xing, Y.; Therien, M. J.; Ratner, M. A.; Borguet, E. Nano Lett. 2014, 14, 5493.

(25) Li, Z.; Smeu, M.; Ratner, M. A.; Borguet, E. J. Phys. Chem. C 2013, 117, 14890.

(26) Li, Z.; Borguet, E. J. Am. Chem. Soc. 2012, 134, 63.

(27) Kiguchi, M.; Takahashi, T.; Kanehara, M.; Teranishi, T.; Murakoshi, K. J. Phys. Chem. C 2009, 113, 9014.

(28) Perrin, M. L.; Verzijl, C. J.; Martin, C. A.; Shaikh, A. J.; Eelkema, R.; Van Esch, J. H.; Van Ruitenbeek, J. M.; Thijssen, J. M.; Van Der Zant, H. S.; Dulić, D. Nat. Nanotechnol. 2013, 8, 282.

(29) Haiss, W.; Wang, C.; Grace, I.; Batsanov, A. S.; Schiffrin, D. J.; Higgins, S. J.; Bryce, M. R.; Lambert, C. J.; Nichols, R. J. Nat. Mater. 2006, 5, 995.

(30) Meisner, J. S.; Kamenetska, M.; Krikorian, M.; Steigerwald, M. L.; Venkataraman, L.; Nuckolls, C. Nano Lett. 2011, 11, 1575.

(31) Gillemot, K.; Evangeli, C.; Leary, E.; La Rosa, A.; González, M. T.; Filippone, S.; Grace, I.; Rubio-Bollinger, G.; Ferrer, J.; Martín, N.; Lambert, C. J.; Agrait, N. Small 2013, 9, 3812.

(32) Roche, C.; Luo, Q.; Gil-Ramírez, G.; Jiang, H.-W.; Kohn, D. R.; Xiong, Y.; Thompson, A. L.; Anderson, H. L. J. Org. Chem. 2017, 82, 7446.

(33) Parker, C. R.; Leary, E.; Frisenda, R.; Wei, Z.; Jennum, K. S.; Glibstrup, E.; Abrahamsen, P. B.; Santella, M.; Christensen, M. A.;
Della Pia, E. A.; Li, T.; González, M. T.; Jiang, X.; Morsing, T. J.; Rubio-Bollinger, G.; Laursen, B. W.; Nørgaard, K.; van der Zant, H.; Agrait, N.; Nielsen, M. B. J. Am. Chem. Soc. 2014, 136, 16497.

(34) Gonzalez, M. T.; Leary, E.; Garcia, R.; Verma, P.; Herranz, M. A.; Rubio-Bollinger, G.; Martin, N.; Agrait, N. J. Phys. Chem. C 2011, 115, 17973.

(35) Jung, T. A.; Schlittler, R. R.; Gimzewski, J. K. Nature 1997, 386, 696.

(36) Senge, M. O.; MacGowan, S. A.; O'Brien, J. M. Chem. Commun. 2015, 51, 17031.

(37) Arroyo, C. R.; Leary, E.; Castellanos-Gomez, A.; RubioBollinger, G.; González, M. T.; Agrait, N. J. Am. Chem. Soc. 2011, 133, 14313.

(38) Valkenier, H.; Huisman, E. H.; van Hal, P. A.; de Leeuw, D. M.; Chiechi, R. C.; Hummelen, J. C. J. Am. Chem. Soc. 2011, 133, 4930.

(39) Fujimoto, T.; Umekawa, H.; Nishino, N. Chem. Lett. 1992, 21, 37.

(40) Freitag, R. A.; Whitten, D. G. J. Phys. Chem. 1983, 87, 3918.

(41) Hatano, K.; Kawasaki, K.; Munakata, S.; Iitaka, Y. Bull. Chem. Soc. Jpn. 1987, 60, 1985.

(42) Okuno, Y.; Kamikado, T.; Yokoyama, S.; Mashiko, S. J. Mol. Struct.: Theochem 2002, 594, 55.

(43) Ferrer, J.; Lambert, C. J.; García-Suárez, V. M.; Manrique, D. Z.; Visontai, D.; Oroszlany, L.; Rodríguez-Ferradás, R.; Grace, I.; Bailey, S.; Gillemot, K.; et al. New J. Phys. 2014, 16, 093029.

(44) Soler, J.; Artacho, E.; Gale, J.; Garcia, A.; Junquera, J.; Ordejon, P.; Sanchez-Portal, D. J. Phys.: Condens. Matter 2002, 14, 2745.

(45) Perdew, J. P.; Burke, K.; Ernzerhof, M. Phys. Rev. Lett. 1996, 77, 3865.

(46) Anderson, H. L. Chem. Commun. 1999, 2323.

(47) Zotti, L. A.; Kirchner, T.; Cuevas, J.-C.; Pauly, F.; Huhn, T.; Scheer, E.; Erbe, A. Small 2010, 6, 1529.

(48) Quek, S. Y.; Khoo, K. H. Acc. Chem. Res. 2014, 47, 3250.

(49) Li, X.; He, J.; Hihath, J.; Xu, B.; Lindsay, S.; Tao, N. J. Am. Chem. Soc. 2006, 128, 2135.

(50) Nef, C.; Frederix, P. L. T. M.; Brunner, J.; Schönenberger, C.; Calame, M. Nanotechnology 2012, 23, 365201.

(51) Aradhya, S. V.; Meisner, J. S.; Krikorian, M.; Ahn, S.; Parameswaran, R.; Steigerwald, M. L.; Nuckolls, C.; Venkataraman, L. Nano Lett. 2012, 12, 1643.

(52) Mishchenko, A.; Vonlanthen, D.; Meded, V.; Buerkle, M.; Li, C.; Pobelov, I. V.; Bagrets, A.; Viljas, J. K.; Pauly, F.; Evers, F.; Mayor, M.; Wandlowski, T. Nano Lett. 2010, 10, 156.

(53) Xu, B.; Xiao, X.; Tao, N. J. J. Am. Chem. Soc. 2003, 125, 16164. 